\title{
A Comparison between Bright Field and Phase-Contrast Image Analysis Techniques in Activated Sludge Morphological Characterization
}

\author{
D.P. Mesquita, ${ }^{1}$ O. Dias, ${ }^{1}$ A.L. Amaral,${ }^{1,2}$ and E.C. Ferreira ${ }^{1, \star}$ \\ ${ }^{1}$ IBB —Institute for Biotechnology and Bioengineering, Centre of Biological Engineering, Universidade do Minho, \\ Campus de Gualtar, 4710-057, Braga, Portugal \\ ${ }^{2}$ Instituto Superior de Engenharia de Coimbra, Instituto Politécnico de Coimbra, Rua Pedro Nunes, \\ Quinta da Nora, 3030-199 Coimbra, Portugal
}

\begin{abstract}
Different approaches using microscopy image analysis procedures were employed for characterization of activated sludge systems. The approaches varied mainly on the type of visualization and acquisition method used for collection of data. In this context, this study focused on the comparison of the two most common acquisition methods: bright field and phase-contrast microscopy. Images were acquired from seven different wastewater treatment plants for a combined period of two years. Advantages and disadvantages of each acquisition technique and the results are discussed. Bright field microscopy proved to be more simple and inexpensive and provided the best overall results.
\end{abstract}

Key words: activated sludge, image analysis, bright field, phase contrast, aggregates, filaments

\section{INTRODUCTION}

An activated sludge system includes a complex ecosystem composed of different types of microorganisms such as protozoa, metazoa, and filamentous or zoogleal bacteria. A good balance between the different microorganisms is essential to guarantee good settling properties and a clear supernatant (Jenkins et al., 2003). Generally, the evaluation of aerated tanks may be performed by visual inspection under an optical microscope coupled to automated image analysis methods. Activated sludge processes have been increasingly monitored through microscopy observations for aggregate contents and morphology and determination of protruding filamentous bacteria content (da Motta et al., 2001b; Jenné et al., 2006). Subsequently, the gathered image analysis information is correlated with the sludge settling abilities (Ganczarczyk, 1994; Grijspeerdt \& Verstraete, 1997; Banadda et al., 2005) to assess biomass morphology changes (Jenné et al., 2003) and to monitor bulking events in pilot plants (da Motta et al., 2001a, 2001b; Jenné et al., 2004, 2007; Amaral \& Ferreira, 2005).

Use of automated image analysis applications in analysis of activated sludge has increased in recent years, with two image acquisition methods standing out: phase-contrast

Received April 8, 2009; accepted December 15, 2009

${ }^{\star}$ Corresponding author. E-mail: ecferreira@deb.uminho.pt microscopy as proposed in the works of Cenens et al. (2002) and Jenné et al. $(2006,2007)$ among others, and bright field microscopy as in the works of da Motta et al. (2001a, 2001b), Amaral and Ferreira (2005), and Mesquita et al. $(2009 a, 2009 b)$. In comparison, bright field microscopy is the cheapest and simplest method to examine activated sludge, whereas phase-contrast microscopy requires more expensive equipment and a more skilled operator. Furthermore, the nature of phase-contrast microscopy causes the aggregate borders to become ill-defined as the object's halo hinders the assessment of their boundaries. However, this method presents, at least in theory, the advantage of a more precise determination of the protruding filamentous bacteria content. The high transparency of the filamentous bacteria poses a contrast problem in bright field microscopy acquisition, which is opposite of the clear filament/dark background distinction in phase-contrast microscopy. Therefore, studies have been performed using bright field acquisition methods to survey the aggregated biomass and phasecontrast acquisition for assessment of filamentous bacteria (Amaral, 2003; Abreu et al., 2007; Costa et al., 2007).

Based on the reported advantages and disadvantages of both methodologies, the present work aims to survey the aggregates and protruding filamentous bacteria contents of activated sludge using image analysis procedures for bright field and phase-contrast microscopy. The best acquisition method for activated sludge characterization was determined by monitoring the activated sludge of seven different wastewater treatment plants for a period of two years. 


\section{Material and Methods}

Activated sludge samples for each acquisition methodology study were collected from the aeration basins of seven wastewater treatment plants, treating domestic effluents for a period of two years. Plants were located in the north of Portugal. A total of 128 samples were analyzed, and for each the biomass content in terms of total suspended solids (TSS) was determined by weighing (APHA et al., 1989). A total of 200 images for each sample was subsequently acquired and processed, in both phase-contrast and bright field microscopy, to estimate the microbial aggregate and protruding filamentous bacteria contents by image analysis techniques.

\section{Image Acquisition}

For each sample, a volume of $25 \mu \mathrm{L}$ was placed on a slide and covered with a $20 \mathrm{~mm} \times 20 \mathrm{~mm}$ cover slip for visualization and image acquisition in bright field and phasecontrast microscopy. Sample deposition was performed by means of a calibrated micropipette with a tip that allowed passage of the largest aggregates. A total of 200 images (divided through three replicate slides) were acquired per sample to obtain significant data for both acquisition methods and to minimize sampling errors. Image acquisition of the aggregates and filaments on each slide was obtained by three horizontal passages at one-quarter, one-half, and threequarters of the slide. In all horizontal passages an image was acquired at each field length. Around 20 images were obtained for each slide. This method overcomes deposition of nonuniform aggregates and filaments on the slide.

\section{Bright Field Microscopy}

Images were acquired with a Leitz Laborlux $S$ optic microscope (Leitz, Wetzlar), with $100 \times$ magnification, coupled to a Zeiss AxioCam (Zeiss, Oberkochen). Image acquisition was performed in $1,300 \times 1,030$ pixels and 8 -bit format through the commercial software Axio Vision 3.1 (Zeiss, Oberkochen).

\section{Phase-Contrast Microscopy}

Images were acquired with a Diaphot 300 microscope (Nikon Corporation, Tokyo) with $100 \times$ magnification, coupled to a Sony CCD AVC-D5CE (Sony, Tokyo) grayscale video camera. The images were acquired in $768 \times 576$ pixels and 8-bit format by a Data Translation DT 3155 (Data Translation, Marlborough, MA) frame grabber using the commercial software package Image Pro Plus (Media Cybernetics, Silver Spring, MD).

To compare both acquisition methods, calibration from pixels to the metric unit dimension was performed by means of a micrometer slide.

\section{Image Processing and Analysis Methodology}

The aggregated and filamentous bacteria content and morphological descriptors were determined through the use of image processing and analysis programs adapted from previous routines first developed by Amaral (2003) and afterward by Amaral and Ferreira (2005) in MATLAB 7.3 (The MathWorks, Inc., Natick, MA) language. Therefore, two different image processing routines were used, one dedicated to bright field images and the other to phase-contrast images. The main stages of these programs comprise the image pretreatment, segmentation, and debris elimination steps. Finally, the aggregated and protruding filamentous biomass binary images were saved for morphological characterization of the activated sludge in the image analysis step. A schematic description of the image processing programs is presented in Figure 1.

A more detailed description of the image processing methods is presented below.

Pretreatment. The image preprocessing stage depends on the enhancement of the grayscale images by background determination (bright field and phase-contrast images) and background removal (bright field images). In this stage, the original image is first divided by a background image to minimize background light differences. The aggregates and filaments are further enhanced by using local histogram equalization to improve the contrast of the boundaries.

Segmentation. This stage consists primarily in segmentation of the aggregates and filaments by the determination and simultaneous use of aggregate boundary and core images. The boundary grayscale image is determined by the difference between the dilated and eroded images (of the pretreatment resulting image). The binary boundary image is obtained by segmenting the resulting image by a predefined 0.3 built-in threshold level. The binary core image is obtained by segmenting the pretreatment resulting image by a predefined 0.3 built-in threshold level. Finally, a morphological opening (disk of radius 5) and reconstruction allow for the final aggregate binary image to be determined.

With respect to the filament segmentation, the aggregate binary image is used as a mask to eliminate the aggregates from the original grayscale image, which is next segmented by a $92.5 \%$ percentile based threshold level. A series of erosion and dilation procedures on the aggregate binary image is then performed to enhance filament identification. Next, a predefined 0.2 built-in threshold level allows the determination of a filament marker image that is later used as a mask to reconstruct the filaments more accurately. Finally, a gyration radius based procedure is implemented to discard small filamentous-like debris by the use of a 1.2 cut-off value (Pons \& Vivier, 1999).

Debris elimination. The elimination of residual aggregates (smaller than $3.5 \mu \mathrm{m}$ in diameter) and debris is performed by third-order erosion and reconstruction operations, and all the aggregates cut off by the image boundaries are removed. 


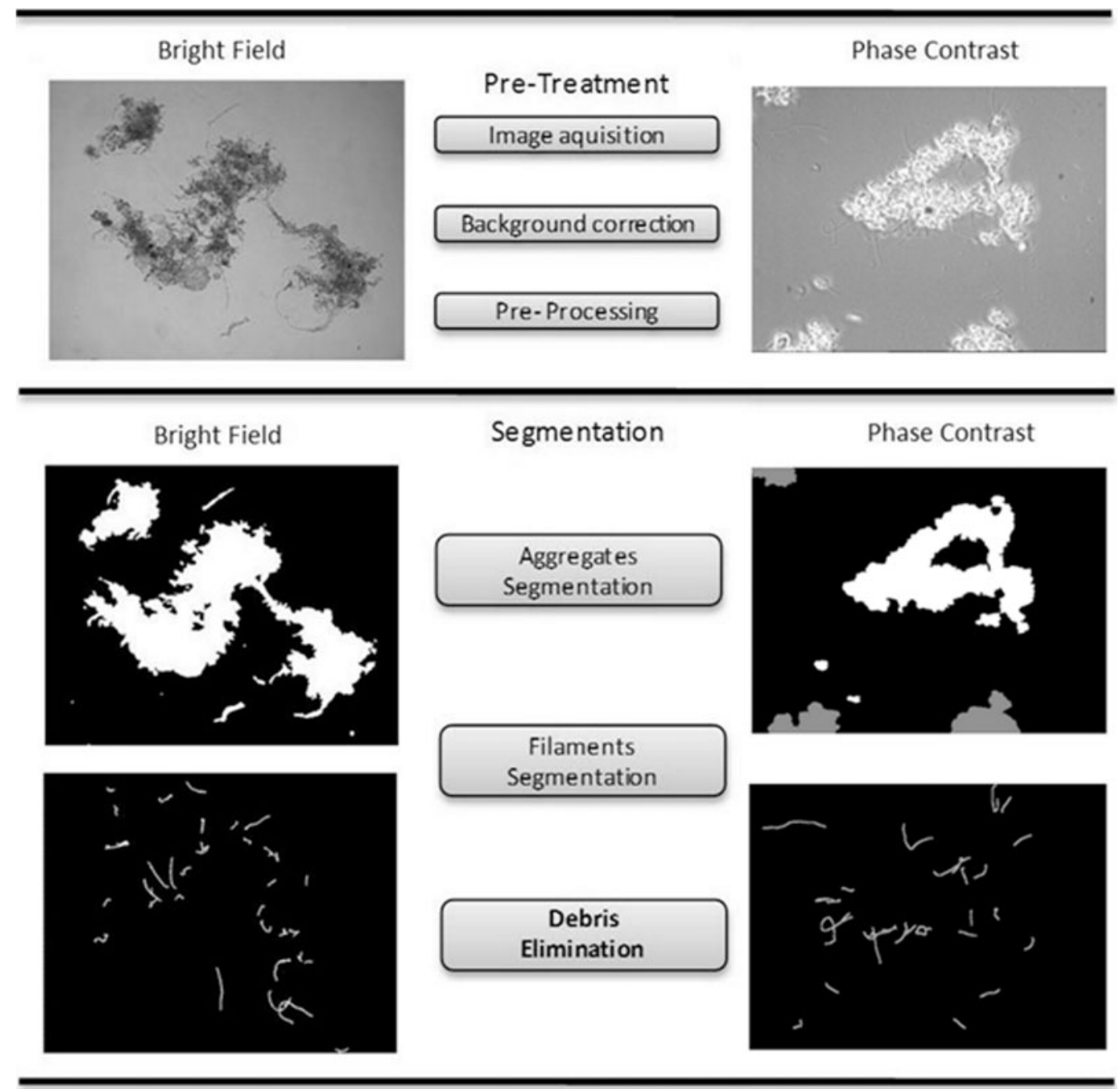

Figure 1. Schematic representation of the bright field and phase-contrast image processing programs.

\section{Morphological Parameters}

Following the image processing step, the recognized aggregated and filamentous bacteria from the collected images were analyzed to individually characterize them in terms of the most relevant morphological parameters described below. To select the best acquisition method, image analysis parameters related to aggregate and filament contents were determined, either directly from the image analysis program or in association with the sludge physical properties for the whole set of 128 samples.

The individual aggregate area (Area) was determined as the pixel sum of each aggregate projected surface calibrated to metric units by a calibration factor $F_{C a l}\left(\mu \mathrm{m}\right.$ pixel $\left.{ }^{-1}\right)$ determined by the use of a micrometer slide. The filaments individual length (FL) was determined according to Walsby and Avery (1996), with $N_{T h n}$ as the pixel sum of each thinned filament, $N_{\text {int }}$ as the number of filament intersections, and factor 1.1222 used to average the different measuring angles within the image. Once again the obtained values were calibrated to metric units by the use of the $F_{C a l}$ $\left(\mu \mathrm{m}\right.$ pixel $\left.^{-1}\right)$ calibration factor:

$$
F L=\left(N_{\text {Thn }}+N_{\text {int }}\right) \times 1.1222 \times F_{\text {cal }} .
$$

Next, the total aggregate area per volume (TA/Vol) and total filament length per volume (TL/Vol) were determined for each replicate, respectively, as the sum of all aggregate areas per unit of volume and the sum of all filament lengths per unit of volume. Finally, for each sample, the average value of the three replicates was then determined. Furthermore, the total filament length per total aggregate area (TL/TA) was also determined alongside the total filament length per total suspended solids (TL/TSS). This characterized the aggregate and filament dynamics considered by Mesquita et al. (2009b) as the most relevant for sludge settling.

To compare both bright field and phase-contrast acquisition methods, the $F$ test two-sample for variances was 

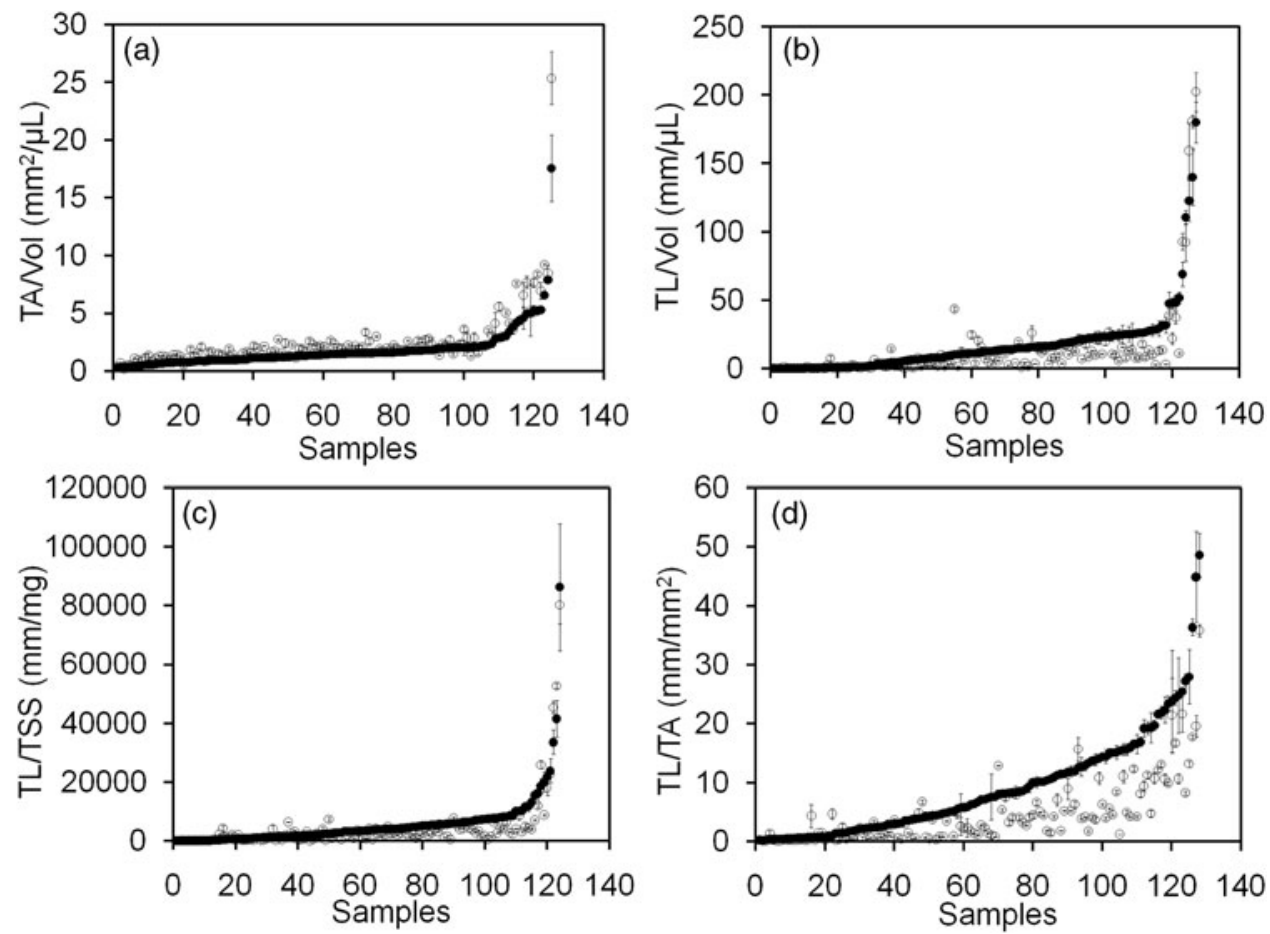

Figure 2. Bright field (•) and phase-contrast (O) results for (a) TA/Vol $\left(\mathrm{mm}^{2} / \mu \mathrm{L}\right),(\mathbf{b}) \mathrm{TL} / \mathrm{Vol}(\mathrm{mm} / \mu \mathrm{L}),(\mathbf{c}) \mathrm{TL} / \mathrm{TSS}$ $(\mathrm{mm} / \mathrm{mg})$, and $(\mathbf{d}) \mathrm{TL} / \mathrm{TA}\left(\mathrm{mm} / \mathrm{mm}^{2}\right)$ considering 128 samples plotted in order of ascending bright field values.

applied to the image analysis results using an $\alpha$-value of 0.05 (95\% confidence). The $F$ critical, $f$-, and $p$-values of the $F$-test for the studied 128 different samples after normalization by the mean values of both methods were determined. The $f$-and $p$-values were then compared with the $F$ critical and $\alpha$-values. For an $f<F$ critical and $p>\alpha$, the variances of both methods can be considered equal.

\section{Results And Discussion}

\section{Direct Sample Comparison}

Bright field and phase-contrast methods were evaluated considering the parameters of total filament length and total aggregate area. These are suitable to represent protruding filamentous bacteria and aggregated biomass contents. Furthermore, the TL/TA and TL/TSS parameters, characterizing the aggregate and filament dynamics, were also compared due to their relevance for assessment of sludge settling properties. For a clearer distinction between the values obtained for each acquisition method, the results for the above parameters were plotted in order of ascending bright field values (Fig. 2).

Results revealed relationships between both acquisition methods with respect to the four studied parameters and helped to establish the main differences throughout the dataset range (Fig. 2). Aggregated biomass content values
(Fig. 2a), in terms of TA/Vol, obtained by phase contrast were systematically higher than for bright field methods. Over $90 \%$ of the dataset was higher for the phase-contrast method. The full dataset revealed an average 38.8\% higher $\mathrm{TA} / \mathrm{Vol}$ values for phase-contrast acquisition (average $1.829 \mathrm{~mm}^{2} / \mu \mathrm{L}$ for bright field and $2.538 \mathrm{~mm}^{2} / \mu \mathrm{L}$ for phase contrast). Given the poorer representation of the object boundaries in phase-contrast microscopy, it seems reasonable to conclude that the phase-contrast method overestimated the aggregated biomass contents.

With respect to the protruding filamentous bacteria content (Fig. 2b), in terms of TL/Vol, for $75 \%$ of the dataset, there is an overestimation for bright field results when compared to phase contrast. This resulted in an average $23.9 \%$ lower $\mathrm{TL} / \mathrm{Vol}$ value for phase-contrast acquisition (average $17.318 \mathrm{~mm} / \mu \mathrm{L}$ for bright field and $13.187 \mathrm{~mm} / \mu \mathrm{L}$ for phase contrast). Given the better filament/ background contrast obtained by phase-contrast microscopy, these results were not expected. However, they can be explained by the fact that most of the activated sludge samples analyzed in this work presented short protruding filamentous bacteria. In this sense, the visible filament length in phase contrast would be shortened by the halo of the aggregates, which is a pressing matter in most of the studied activated sludge, presenting low (and short) protruding filamentous bacteria, as can be seen in Figure 2b. When the analysis fell solely on samples presenting long protruding filaments (TL/ $\mathrm{Vol}>50 \mathrm{~mm} / \mu \mathrm{L}$, for samples 123 to 

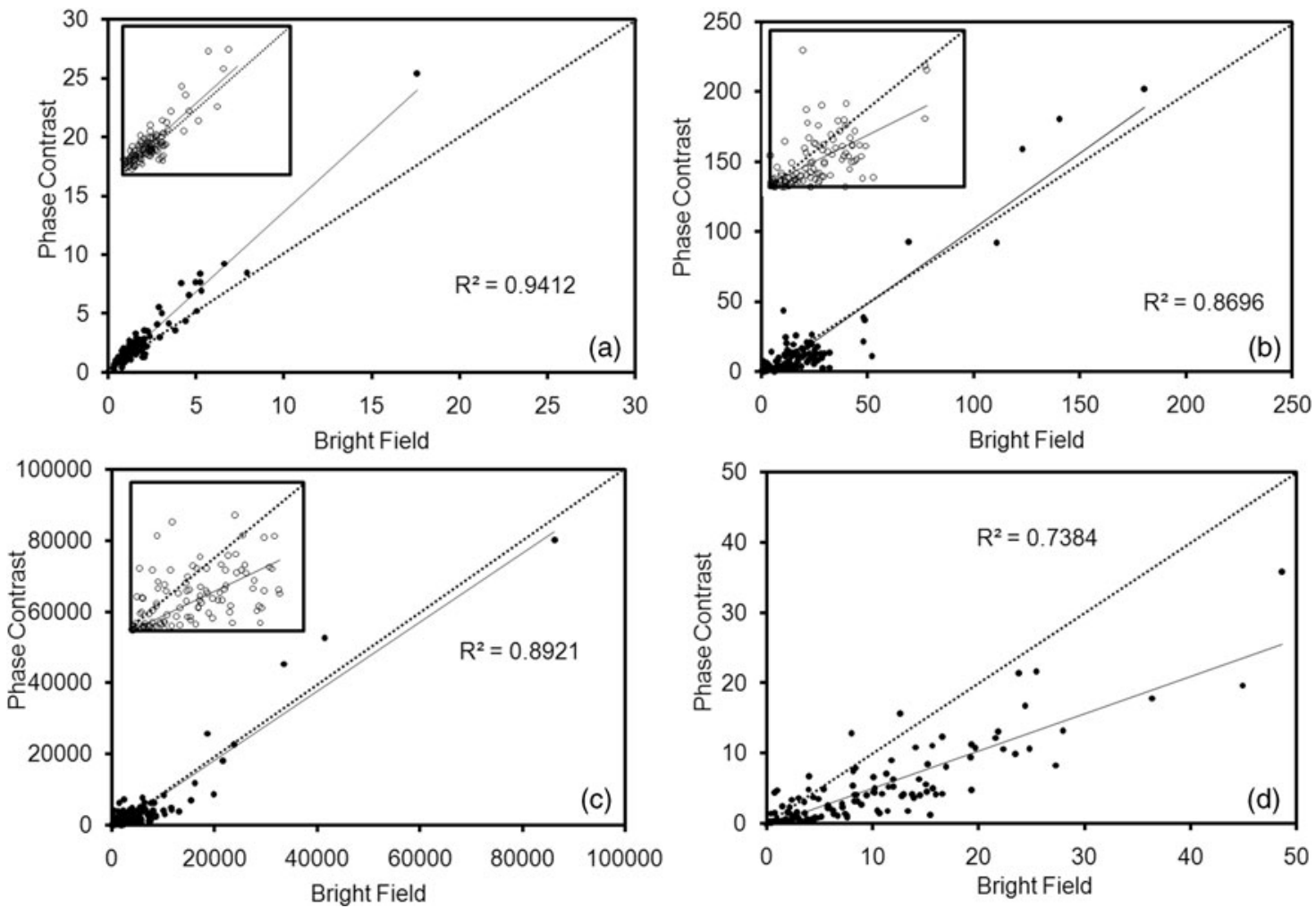

Figure 3. Bright field and phase-contrast regressions for (a) TA/Vol $\left(\mathrm{mm}^{2} / \mu \mathrm{L}\right)$, (b) TL/Vol (mm/ $\left./ \mathrm{L}\right)$, (c) TL/TSS $(\mathrm{mm} / \mathrm{mg})$, and $(\mathbf{d}) \mathrm{TL} / \mathrm{TA}\left(\mathrm{mm} / \mathrm{mm}^{2}\right)$. In panels $\mathbf{a}-\mathbf{c}$ a window is included representing the Log-Log representation for clarity purposes.

128 in Fig. 2b), however, a clearer correspondence could be found between the two methodologies. As a matter of fact, the TL/Vol determined by the bright field methodology on these samples were only $8.6 \%$ on average lower than the phase-contrast methodology results (average $112.61 \mathrm{~mm} / \mu \mathrm{L}$ for the bright field and $123.16 \mathrm{~mm} / \mu \mathrm{L}$ for the phase contrast).

Schuler and Jassby (2007) showed that expressing filament content per mass (TL/TSS) is probably the most useful way for comparing filament content data from different studies and/or from samples with different biomass concentrations. Concentrations can vary greatly from one system to the other, and this approach normalizes filament content to biomass. Regarding this parameter behavior (Fig. 2c) with biomass normalization, a similar trend between bright field and phase-contrast methods arises when compared to the TL/Vol analysis. This results in an underestimation for phase-contrast results when compared to bright field in $75 \%$ of the dataset. Phase-contrast methods for the TL/TSS ratio also underestimated TL/TSS ratio values an average $24.2 \%$ regarding bright field acquisition (average 5,605.1 mm/mg for bright field and 4,246.7 mm/mg for phase contrast). Given the constraints of phase contrast on accurately determining the short protruding filamentous bacteria, an analysis was performed focusing on the long protruding filaments (TL/TSS $>20,000 \mathrm{~mm} / \mathrm{mg}$, for samples 123 to 128 in Fig. 2c). As observed for the TL/Vol analysis, a much higher similarity was found between both methods and only a slight overestimation $(0.6 \%$ on average) was found for the phase-contrast method (average $37,812 \mathrm{~mm} / \mathrm{mg}$ for bright field and $38,025 \mathrm{~mm} / \mathrm{mg}$ for phase contrast).

The difference between bright field and phase-contrast acquisition is also quite clear when observing filament length per aggregate area ratio (Fig. 2d). Systematically (over $90 \%$ of the dataset) higher TL/TA values were obtained for the bright field method. Phase contrast underestimates by an average $50 \%$ the TL/TA values obtained by bright field microscopy (average $9.084 \mathrm{~mm} / \mathrm{mm}^{2}$ for bright field and $4.545 \mathrm{~mm} / \mathrm{mm}^{2}$ for phase contrast) due to the low filament recognition and aggregate area overestimation.

\section{Regression Analysis}

Figure 2 shows a clear relationship between the results for bright field and phase-contrast microscopy acquisition. The correlation between bright field and phase-contrast results was then studied to help establish the best acquisition method for both aggregated and protruding filamentous bacteria. Figure 3 represents the obtained correlations be- 
tween bright field and phase-contrast methods. Figure 3a shows a satisfactory correlation coefficient of $0.9702\left(R^{2}\right.$ of 0.9412) for the TA/Vol between bright field and phasecontrast assessment. Once again, it was possible to conclude that the phase-contrast method overestimated the aggregate detection with respect to the bright field acquisition method. Furthermore, the trend line for the TA/Vol estimation points toward a global overestimation around $36.3 \%$, quite close to the $38.8 \%$ obtained in the direct comparison analysis.

With respect to recognition of the protruding filamentous bacteria (Fig. 3b), the correlation of $0.9325\left(R^{2}\right.$ of 0.8696) was not as satisfactory as for the TA/Vol analysis, but absolute values between the two methods were similar considering the global overestimation of $7.9 \%$ for the phase-contrast results. The higher dispersion (lower correlation coefficient) between the two methods can be explained, as mentioned above, because phase contrast likely underestimated short protruding filaments, thus shifting the low TL/Vol samples away from the global trend line. The regression equation seems to point toward a $5.52 \mathrm{~mm} / \mu \mathrm{L}$ underestimation with the phase-contrast method when compared to the bright field average of $17.32 \mathrm{~mm} / \mu \mathrm{L} \mathrm{TL} / \mathrm{Vol}$ value. This represents an underestimation of $31.9 \%$ in this low filament content range. However, a reasonable correspondence was observed between the two methods as indicated by the low $8.6 \%$ overestimation of high TL/Vol contents with phase contrast in direct comparison analysis and the slight $7.9 \%$ overestimation in the correlation analysis. The reason may be explained by the high importance of the larger TL/Vol samples with respect to the correlation determination by the least-squares method. The lower values on larger filament content of the bright field method balances, to some extent, the larger values obtained for smaller contents. It should be noted that, in samples with larger filament content, the advantage of phase-contrast recognition was less hindered by the ill-defined aggregate borders leading to a more precise (and higher) filament determination. Therefore, the correspondence found between the two methods indicated that bright field was indeed advantageous over phase-contrast acquisition on well-balanced activated sludge systems given that it does not lead to overall significant errors with respect to determination of the protruding filamentous bacteria. However, care should be taken in analyzing the results of bright field acquisition from activated sludge with very high filamentous contents.

Regarding the TL/TSS ratio results (Fig. 3c), a correlation coefficient of $0.9445\left(R^{2}\right.$ of 0.8921$)$ was obtained. Absolute values for both samples were again similar considering the global underestimation of $3.1 \%$ for the phasecontrast results. Once more, the dispersion found between the two methods could be explained by the propensity of phase contrast to underestimate the short protruding filaments, thus shifting the low TL/TSS samples away from the global trend line. The regression equation indicates a $1,200.4 \mathrm{~mm} / \mathrm{mg}$ underestimation with phase contrast, which, relating to the bright field average of $5,605.1 \mathrm{~mm} / \mathrm{mg} \mathrm{TL} /$
Table 1. $f$ - and $p$-Values Obtained by the F-Test Statistical Analysis $(\alpha=0.05)$ Regarding the Normalized Aggregates and Filaments Contents as Determined by Bright Field and Phase-Contrast Methodologies.

\begin{tabular}{lcccc}
\hline Variables & TA/Vol & TL/Vol & TL/TSS & TL/TA \\
\hline$f$-value & 1.024 & 2.304 & 1.828 & 1.525 \\
$p$-value & 0.447 & $1.84 \times 10^{-6}$ & $4.20 \times 10^{-4}$ & 0.009 \\
\hline
\end{tabular}

Vol, represents an underestimation of $21.4 \%$ in this low filament content range. Nonetheless, a good correspondence between the two methods could be found as shown by the slight $3.1 \%$ underestimation with phase contrast and the proximity to the $0.6 \%$ high TL/TSS content underestimation in the direct comparison analysis. The reason for this is directly related to the protruding filamentous bacteria estimation differences found in the two methods. The same caution must be applied in the TL/TSS ratio as in the TL/Vol assessment.

A considerable dispersion between bright field and phase-contrast assessments was detected and a poor 0.8593 correlation coefficient $\left(R^{2}\right.$ of 0.7384$)$ for the TL/TA ratio was achieved (Fig. 3d). This result might be due to the cumulative sum of impreciseness both in terms of TL and TA resulting in a strong underestimation of this parameter in phase-contrast acquisition. This conclusion is further emphasized by the 0.53 slope obtained. This resulted in a global $47 \%$ underestimation (regression slope of 0.53 ) of the TL/TA ratio by phase contrast, which is in accordance with the 50\% reduction in the direct comparison analysis.

\section{Statistical Analysis}

To understand the relationship between bright field and phase-contrast acquisition, the $F$-test two sample for variances was performed for an $\alpha$-value of 0.05 (95\% confidence). Table 1 presents the $f$-values and $p$-values of the statistical analysis comparing the normalized image analysis results for both bright field and phase contrast. A total of 128 samples for each method was analyzed resulting in 127 degrees of freedom and an F critical value of 1.34.

Taking into consideration the $f$-value of 1.024 (lower than the $F$ critical value of 1.34 ) and the $p$-value of 0.447 (larger than the $\alpha$-value of 0.05 ) regarding the TA/Vol, it seems clear that both methods presented similar variances and, therefore, are statistically similar. Furthermore, these results were in accordance with the satisfactory $R^{2}$ value of 0.9412 obtained in the regression analysis.

On the contrary the TL/Vol, TL/TSS, and TL/TA statistical analysis had $f$-values higher than the $F$ critical value of 1.34. This indicated that the sample variances for these parameters were statistically different, and therefore the methods can be considered as unrelated. The same conclu- 
Table 2. $f$ - and $p$-Values Obtained by the F-Test Statistical Analysis $(\alpha=0.05)$ Regarding the Two Normalized TL/Vol and TL/TSS Studied Ranges, as Determined by Bright Field and Phase-Contrast Methodologies.

\begin{tabular}{lccccc}
\hline & \multicolumn{2}{c}{ TL/Vol } & & \multicolumn{2}{c}{ TL/TSS } \\
\cline { 2 - 3 } \cline { 5 - 6 } $\begin{array}{c}c 50 \\
\text { Variables }\end{array}$ & $\begin{array}{c}>50 \\
(\mathrm{~mm} / \mu \mathrm{L})\end{array}$ & & $\begin{array}{c}<2,000 \\
(\mathrm{~mm} / \mu \mathrm{L})\end{array}$ & & $\begin{array}{c}>20,000 \\
(\mathrm{~mm} / \mathrm{mg})\end{array}$ \\
\hline$f$-value & 1.571 & 1.139 & & 1.698 & 1.241 \\
F critical & 1.352 & 6.388 & & 1.352 & 6.388 \\
$p$-value & 0.007 & 0.451 & & 0.002 & 0.420 \\
\hline
\end{tabular}

sion can also be attained regarding the obtained $p$-values, which were smaller than the statistical significance value (0.05). These results seem to corroborate the previous findings of a lower correlation between the bright field and phase-contrast methods for the TL/Vol, TL/TSS, and TL/TA.

A second $F$-test two-sample was also performed for an $\alpha$-value of 0.05 ( $95 \%$ confidence) regarding the samples with high $(\mathrm{TL} / \mathrm{Vol}>50 \mathrm{~mm} / \mu \mathrm{L}, \mathrm{TL} / \mathrm{TSS}>20,000 \mathrm{~mm} / \mathrm{mg})$ and low (TL/Vol $<50 \mathrm{~mm} / \mu \mathrm{L}$, TL/TSS $<20,000 \mathrm{~mm} / \mathrm{mg}$ ) filamentous contents (Table 2). The six samples total for the $\mathrm{TL} / \mathrm{Vol}>50 \mathrm{~mm} / \mu \mathrm{L}$ and TL/TSS $>20,000 \mathrm{~mm} / \mathrm{mg}$ analyses resulted in 5 degrees of freedom and consequently of an $F$ critical value of 6.388 , whereas the 121 samples total for the TL/Vol $<50 \mathrm{~mm} / \mu \mathrm{L}$ and TL/TSS $<20,000 \mathrm{~mm} / \mathrm{mg}$ analyses resulted in 120 degrees of freedom and consequently an F critical value of 1.352 .

For the TL/Vol parameter of high filamentous content samples (TL/Vol $>50 \mathrm{~mm} / \mu \mathrm{L}$ ), both methods had similar variances as shown by the $f$-value of 1.139 (lower than the $F$ critical value of 6.388) and $p$-value of 0.451 (larger than the $\alpha$-value of 0.05). Therefore, it could be established that for the high TL/Vol range both methods are statistically similar, as it was hypothesized in the previous direct sample comparison analysis. With respect to the low filamentous content samples (TL/Vol $<50 \mathrm{~mm} / \mu \mathrm{L}$ ), the $f$-value of 1.571 (higher than the $F$ critical value of 1.352) and $p$-value of 0.007 (smaller than the $\alpha$-value of 0.05 ) led to the conclusion that the two methods were statistically different in the low
TL/Vol range. Once again, these results corroborated the conclusions of the direct sample comparison analysis.

A similar result was also observed for the TL/TSS parameter. In high filamentous content samples (TL/TSS > $20,000 \mathrm{~mm} / \mathrm{mg}$ ), the $f$-value of 1.241 (lower than the $F$ critical value of 6.388) and $p$-value of 0.420 (larger than the $\alpha$-value of 0.05$)$ point to statistical similarity of the two methods. In low filamentous content samples (TL/TSS $<$ $20,000 \mathrm{~mm} / \mathrm{mg}$ ), the $f$-value of 1.698 (higher than the $F$ critical value of 1.352) and $p$-value of 0.002 (smaller than the $\alpha$-value of 0.05 ) point to the opposite conclusion regarding the low TL/TSS range. As above, these results also corroborated the conclusions of the direct sample comparison analysis.

\section{Accuracy Analysis}

To determine the accuracy of both methods, the average of the TA/Vol, TL/Vol, TL/TA, and TL/TSS for all samples, the average of the standard deviations for all samples, and the average error percentages for all samples were determined (Table 3). The parameter and their standard deviations were determined as the average of the 128 samples for that parameter. The accuracy error percentage was determined as the ratio between the average parameter standard deviation and the average parameter value, multiplied by 100 .

Accuracy error percentages for all parameters for both methods showed no significant variations, although a slight advantage for the phase-contrast method was apparent, with exception of the TL/TA parameter. These results indicate that both methodologies are almost equivalent. For both phase-contrast and bright field methods, the accuracy error percentage remained around $10 \%$ of the average value and within a window of $2 \%$. Only the TL/TSS difference was more significant (10.74\% against $7.18 \%)$. The TL/Vol accuracy error percentage for the two methods was much closer (11.06\% against $9.70 \%$ ), and this difference may be partly explained by a higher error associated with the TSS measurement regarding the bright field samples.

\section{Robustness Analysis}

This study also addressed the robustness evaluation of both methods in terms of aggregated and protruding filamentous

Table 3. Average Values, Average Standard Deviation, and Accuracy Error Percentage Values Regarding the Aggregates and Filaments Contents as Determined by Bright Field (BF) and Phase-Contrast (PC) Methodologies.

\begin{tabular}{|c|c|c|c|c|c|c|c|c|}
\hline \multirow[b]{2}{*}{ Variables } & \multicolumn{2}{|c|}{$\mathrm{TA} / \mathrm{Vol}\left(\mathrm{mm}^{2} / \mu \mathrm{L}\right)$} & \multicolumn{2}{|c|}{$\mathrm{TL} / \mathrm{Vol}(\mathrm{mm} / \mu \mathrm{L})$} & \multicolumn{2}{|c|}{$\mathrm{TL} / \mathrm{TSS}(\mathrm{mm} / \mathrm{mg})$} & \multicolumn{2}{|c|}{$\mathrm{TL} / \mathrm{TA}\left(\mathrm{mm} / \mathrm{mm}^{2}\right)$} \\
\hline & $\mathrm{BF}$ & PC & $\mathrm{BF}$ & PC & $\mathrm{BF}$ & PC & $\mathrm{BF}$ & PC \\
\hline Average values & 1.829 & 2.538 & 17.318 & 13.187 & 5605.1 & 4246.7 & 9.084 & 4.545 \\
\hline Average standard deviation & 0.186 & 0.229 & 1.914 & 1.279 & 602.2 & 304.9 & 0.759 & 0.415 \\
\hline Average error \% & 10.18 & 9.02 & 11.06 & 9.70 & 10.74 & 7.18 & 8.36 & 9.13 \\
\hline
\end{tabular}


Table 4. Number and Percentage of Images Treated with Default and Changed Values during the Processing Step for Bright Field and Phase-Contrast Programs for Both Aggregates and Protruding Filamentous Bacteria Detection.

\begin{tabular}{lcccc}
\hline & \# Default & \# Changed & $\%$ Default & $\%$ Changed \\
\hline Bright field & & & & \\
Aggregates & 33,168 & 0 & $100 \%$ & $0 \%$ \\
$\quad$ Filaments & 32,000 & 1168 & $96 \%$ & $4 \%$ \\
Phase contrast & & & & \\
$\quad$ Aggregates & 33,230 & 694 & $98 \%$ & $2 \%$ \\
Filaments & 29,309 & 4615 & $86 \%$ & $14 \%$ \\
\hline
\end{tabular}

bacteria detection by the developed image processing programs. Default values for the detection thresholds of aggregates and filaments, loaded in the beginning of both programs, were analyzed throughout monitoring of the seven wastewater treatment plants. After each processing step, and to avoid inaccurate assessment, the final labeled images were scrutinized by visual inspection. Thus, during the monitoring period, the final binary aggregate and filament images that were not in accordance with the original images were reprocessed, with minor adjustments on the detection thresholds. Table 4 displays the recognition results for both bright field and phase-contrast methods regarding the detection of aggregates and protruding filaments, with default and changed values.

Data in Table 4 point out the high robustness for detection of both aggregate and filamentous bacteria with the default values provided from the bright field and phasecontrast programs. Filaments detection proved to be most prone to errors with $4 \%$ and $14 \%$ adjustments needed for bright field and phase-contrast methods, respectively. This result can be explained by the small width of the filaments and higher microscopy focusing dependence. Comparing the two acquisition methods, phase contrast seems to be less robust than bright field leading to a higher percentage of changes in default values for recognition of both aggregates and filamentous bacteria. The robustness of the bright field method was $100 \%$ for aggregate recognition and $96 \%$ for filament recognition. Only $98 \%$ and $86 \%$ robustness values were attained for the same parameters regarding the phasecontrast method.

\section{CONCLUSIONS}

This study demonstrated that, with respect to the protruding filamentous bacteria, bright field acquisition results are similar to the phase-contrast results. However, a clear discrepancy between methods in the low filament samples was found. This may be due to the existence, in phase-contrast acquisition, of a halo surrounding the aggregates. This hinders their correct determination by this method. If the halo of an aggregate is too bright then the part of the filament that falls within the halo area may not be visible as it is quite thin. Furthermore, it may not be simple to discern the border between the halo and the actual aggregate, making it difficult to estimate the length of the filament encompassed by the aggregate's halo. In assessment of the aggregated biomass content, both methods are statistically similar, although with a clear overestimation of the aggregate area for the phase-contrast acquisition. An accuracy analysis also demonstrated no significant variations for both methods. Although both methods provided very high robustness, bright field microscopy surpassed phase-contrast results in this matter. Considering the advantages and disadvantages of each acquisition method, bright field microscopy proved to be a more simple and inexpensive method that provided the best overall results.

\section{ACKNOWLEDGMENTS}

The authors acknowledge the financial support to D.P.M. and O.D. through the grant SFRH/BD/32329/2006 and the project POCI/AMB/57069/2004, respectively, provided by Fundação para a Ciência e Tecnologia (Portugal). The authors express their gratitude to AGERE (Empresa de Águas, Efluentes e Resíduos de Braga, Portugal-EM).

\section{REFERENCES}

Abreu, A.A., Costa, J.C., Araya-Kroff, P., Ferreira, E.C. \& Alves, M.M. (2007). Quantitative image analysis as a diagnostic tool for identifying structural changes during a revival process of anaerobic granular sludge. Water Res 41, 1473-1480.

Amaral, A.L. (2003). Image analysis in biotechnological processes: Applications to wastewater treatment. Ph.D. Thesis, Braga, Portugal. Available at http://hdl.handle.net/1822/4506.

Amaral, A.L. \& Ferreira, E.C. (2005). Activated sludge monitoring of a wastewater treatment plant using image analysis and partial least squares regression. Anal Chim Acta 544, 246-253.

APHA, AWWA \& WPCF. (1989). Standard Methods for the Examination of Water and Wastewater, 17th Ed. Washington, D.C.: American Public Health Association.

Banadda, E.N., Smets, I.Y., Jenné, R. \& Van Impe, J.F. (2005). Predicting the onset of filamentous bulking in biological wastewater treatment systems by exploiting image analysis information. Bioproc Biosys Eng 27, 339-348.

Cenens, C., Van Beurden, K.P., Jenné, R. \& Van Impe, J.F. (2002). On the development of a novel image analysis technique to distinguish between flocs and filaments in activated sludge images. Water Sci Technol 46(1-2), 381-387.

Costa, J.C., Abreu, A.A., Ferreira, E.C. \& Alves, M.M. (2007). Quantitative image analysis as a diagnostic tool for monitoring structural changes of anaerobic granular sludge during detergent shock loads. Biotechnol Bioeng 98(1), 60-68. 
da Motta, M., Amaral, A.L., Casellas, M., Pons, M.N., Dagot, C., Roche, N., Ferreira, E.C. \& Vivier, H. (2001a). Characterisation of activated sludge by automated image analysis: Validation on full-scale plants. IFAC Computer Applications in Biotechnology, Québec City, Canada, pp. 427-431.

da Motta, M., Pons, M.N. \& Roche, N. (2001b). Automated monitoring of activated sludge in a pilot plant using image analysis. Water Sci Technol 43(7), 91-96.

GANCZARCZYK, J.J. (1994). Microbial aggregates in wastewater treatment. Water Sci Technol 30, 87-95.

Grijspeerdt, K. \& Verstraete, W. (1997). Image analysis to estimate the settleability and concentration of activated sludge. Water Res 31, 1126-1134.

Jenkins, D., Richard, M.G. \& Daigger, G. (2003). Manual on the Causes and Control of Activated Sludge Bulking, Foaming and Other Solids Separation Problems. Boca Raton, FL: Lewis Publishing.

Jenné, R., Banadda, E.N., Gins, G., Deurinck, J., Smets, I.Y., GeEraerd, A.H. \& VAn Impe, J.F. (2006). Use of image analysis for sludge characterisation: Studying the relation between floc shape and sludge settleability. Water Sci Technol 54(1), $167-174$.

Jenné, R., Banadda, E.N., Philips, N. \& Van Impe, J.F. (2003). Image analysis as a monitoring tool for activated sludge prop- erties in lab-scale installations. J Environ Sci Health Part A-Toxic/Hazardous Subs \& Environ Eng 38(10), 2009-2018.

Jenné, R., Banadda, E.N., Smets, I.Y., Deurinck, J. \& Van Impe, J.F. (2007). Detection of filamentous bulking problems: Developing an image analysis system for sludge composition monitoring. Micros Microanal 13, 36-41.

Jenné, R., Banadda, E.N., Smets, I.Y. \& Van Impe, J.F. (2004). Monitoring activated sludge settling properties using image analysis. Water Sci Technol 50(7), 281-285.

Mesquita, D.P., Dias, O., Amaral, A.L. \& Ferreira, E.C. (2009a). Monitoring of activated sludge settling ability through image analysis: Validation on full-scale wastewater treatment plants. Bioprocess Biosyst Eng 32(3), 361-367.

Mesquita, D.P., Dias, O., Dias, A.M.A., Amaral, A.L. \& FerReIrA, E.C. (2009b). Correlation between sludge settleability and image analysis information using partial least squares. Anal Chim Acta 642 (1-2), 94-101.

Pons, M.N. \& Vivier, H. (1999). Biomass quantification by image analysis. Adv Biochem Eng/Biotech 66, 133-184.

Schuler, A.J. \& Jassby, D. (2007). Filament content threshold for activated sludge bulking: Artifact or reality? Water Res 41, 4349-4356.

Walsby, A.E. \& Avery, A. (1996). Measurement of filamentous cyanobacteria by image analysis. J Microbiol Methods 26, 11-20. 